Ann. Zootech., I977, 27 (2), 2II-223.

\title{
Caractéristiques des consommations d'aliments solide et liquide chez la Lapine gestante puis allaitante nourrie ad libitum
}

\author{
Y. REYNE, M. PRUD'HON, Anne-Marie DEBICKI, J. GOUSSOPOUlos \\ avec la collaboration technique de J. Axgevain \\ Station de Physiologie animale, I.N.R.A., \\ L.N.S.A., Place Viala, \\ 34060 Montpellier Cedex (France)
}

\begin{abstract}
Résumé
La fréquence, l'amplitude et la répartition nycthémérale des prises d'aliment et d'eau de boisson ont été enregistrées graphiquement chez des lapines successivement gestantes puis allaitantes. Le dispositif d'enregistrement était celui décrit par PRUD'HON et al. (I972). Deux lots de 4 lapines (allaitement imposé à heure fixe), et 7 lapines (allaitement libre) de race " Néozélandaise blanche " ont été étudiés respectivement au cours de 9 et 8 cycles gestation-lactation.

En dehors de l'évolution bien connue de la consommation et de la production laitière au cours des trois premières semaines de lactation, il est possible de noter : de la mise bas;

- un arrêt presque total de la consommation d'aliment et d'eau la veille ou l'avant-veille

- une consommation nocturne largement prédominante chez les lapines gestantes, quel que soit le stade de gestation;

- une modification de la répartition nycthémérale des prises dès le début de la lactation;

- chez les lapines allaitant à heure fixe, on a une distribution biphasique des consommations, les valeurs maximales étant situées respectivement après la tétée et au cours des heures encadrant l'extinction de la lumière.

Chez les lapines en allaitement libre, la répartition des consommations est plus variable, autant diurne que nocturne.

De plus, l'accroissement de la consommation chez les lapines allaitantes semble dûe essentiellement à l'accroissement de la fréquence des prises solides et à un moindre degré à l'accroissement de leur amplitude en fin de lactation. Ce phénomène est moins marqué et plus variable dans le cas des prises liquides où la variation de l'amplitude des prises joue un rôle plus important.

Enfin, les corrélations entre lait produit et aliment consommé en 24 heures sont élevées. Au contraire, les coefficients de corrélation partielle entre le lait produit et l'aliment consommé dans les deux heures qui suivent la tétée sont très faibles, ce qui suggère que le rétablissement de l'équilibre entre sorties et entrées d'eau et d'énergie ne se fait parfaitement que sur une période de 24 heures ou plus.
\end{abstract}




\section{Introduction}

Les caractéristiques générales de l'ingestion d'aliments solide et liquide chez le Lapin domestique ont été décrites par Chevillard, GasNier et Mayer (I939), PRUd'hon et al. (I972), SANDERSON et VANDERWEElE (I975). Si l'on excepte l'existence d'une cæcotrophie les traits les plus marquants du comportement alimentaire sont la multiplicité des prises d'aliment et l'existence d'un rythme nycthéméral d'ingestion régulier. Toutefois la fréquence des prises, leur amplitude et leur répartition au cours du nycthémère varient avec de nombreux facteurs; l'âge (PRUD'HON et al., I975a), la température ambiante (PRUD'HON, I976), le jeûne, l'injection d'insuline ou de glucose (HöRNICKE et al., I976), les conditions d'abreuvement (PRUD'HON et al., I975b).

Il est également probable que le comportement alimentaire varie en fonction de l'état physiologique de l'animal. C'est pourquoi l'étude de l'évolution des caractéristiques de consommation chez la lapine gestante puis allaitante constitue un modèle intéressant. Celui-ci permet également d'analyser les modifications de comportement survenant lorsque le niveau de consommation varie brutalement du simple au triple.

\section{Matériel et méthodes}

Dans un premier temps nous avons étudié le comportement alimentaire de quatre lapines de race "Néozélandaise blanche " au cours de 9 cycles gestationlactation. Les lapines ont été placées dans le dispositif d'enregistrement graphique des consommations décrit précédemment par PRUD'HON et al. (I972). Elles étaient âgées de 7 à $\mathrm{I} 2$ mois à la mise bas, le numéro de mise bas variant de $\mathrm{I}$ à 3 et le nombre de lapereaux de 2 à II. Les lapines ont été suivies du début de la gestation jusqu'au sevrage des lapereaux trois semaines après la mise bas. Dès la mise bas, les lapereaux ont été séparés de leur mère. Ils n'ont été remis en sa présence pour la tétée qu'une fois par jour pendant ro minutes environ vers 9 heures. On sait en effet que dans des conditions naturelles d'allaitement maternel les lapereaux n'effectuent généralement qu'une seule tétée par jour (ZARrow, DENENBERG et ANDERSON, I965).

I a quantité de lait produite par jour a été estimée par pesée des lapines avant et après la tétée, selon la méthode de LEBAS (I968). Il en a été déduit pour chaque portée la consommation moyenne hebdomadaire par lapereau. Les lapereaux ont été pesés individuellement tous les sept jours, du jour de la mise bas jusqu'au sevrage. Ce premier groupe de lapines constituait le lot à "allaitement contrôlé ".

I'allaitement à heure fixe étant susceptible de modifier le comportement alimentaire des lapines allaitantes, nous avons étudié également un deuxième groupe de 7 lapines ayant libre accès au nid. Ces lapines étaient âgées de 9 à $\mathrm{I} 7$ mois à la mise bas; le numéro de mise bas variait de I à 6 et le nombre de lapereaux de 4 à Ir. Ce groupe constituait le lot à "allaitement libre " ( 8 cycles gestationlactation). Les deux groupes étaient éclairés de 7 heures à 2 I heures. La température du local était maintenue à $20 \pm \mathrm{I}^{\circ} \mathrm{C}$. L'eau de boisson était distribuée à volonté par un abreuvoir à clapet et les animaux disposaient, également à volonté, d'un aliment granulé du commerce dosant $\mathrm{I}_{5} \mathrm{p}$. cent de protéines brutes et $\mathrm{I}_{4} \mathrm{p}$. cent de matières cellulosiques. 


\section{Résultats}

I. - Caractéristiques générales de la consommation d'aliment et d'eau de boisson pendant la gestation et la lactation

Sur la figure I nous avons représenté à titre d'exemple l'évolution de la consommation d'aliment solide en fonction du temps au cours de trois mises bas successives chez une même lapine. Il faut noter une diminution progressive de la consommation en fin de gestation. Cette diminution s'accentue 7 à 8 jours avant



FIG. I. - Evolution de la consommation solide au cours de trois mises bas successives : exemple d'une lapine.

Solid lood intake evolution during three consecutive gestation-lactation cycles: example for one animal.

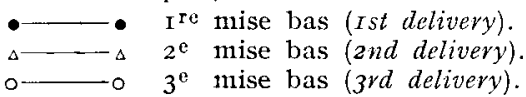

(Lors des deuxième et troisième gestations, la lapine est pendant deux semaines à la fois gestante et allaitante et ces données ont été éliminées).

(During the second and third gestation-lactation cycles, the doe is for two weeks pregnant and lactating at the same time and these data have been eliminated). 
la mise bas puis est suivie d'un pic de consommation vers le quatrième jour avant mise bas. Ce pic est plus ou moins marqué selon les lapines. Il est parfois absent, mais lorsqu'il existe, il se reproduit généralement pour une même lapine au cours de ses mises bas successives. La consommation chute ensuite très fortement. Elle est minimum la veille ou l'avant-veille de la mise bas et peut alors être pratiquement nulle pour certaines lapines. Après la mise bas, la consommation augmente très vite durant les 6-7 premiers jours puis plus lentement ensuite.

La consommation augmente également avec le numéro de mise bas, que ce soit au cours de la gestation ou au cours de la lactation, l'allure générale de la courbe restant à peu près la même.

L'évolution de la consommation d'eau de boisson tout au long de la gestation et de 1a lactation est pratiquement analogue chez une même lapine à celle de 1'aliment solide. Cette consommation évolue également de manière identique à celle de 1'aliment solide au cours des mises bas successives.

La figure 2 fournit un exemple d'analyse plus détaillée des principales carac-
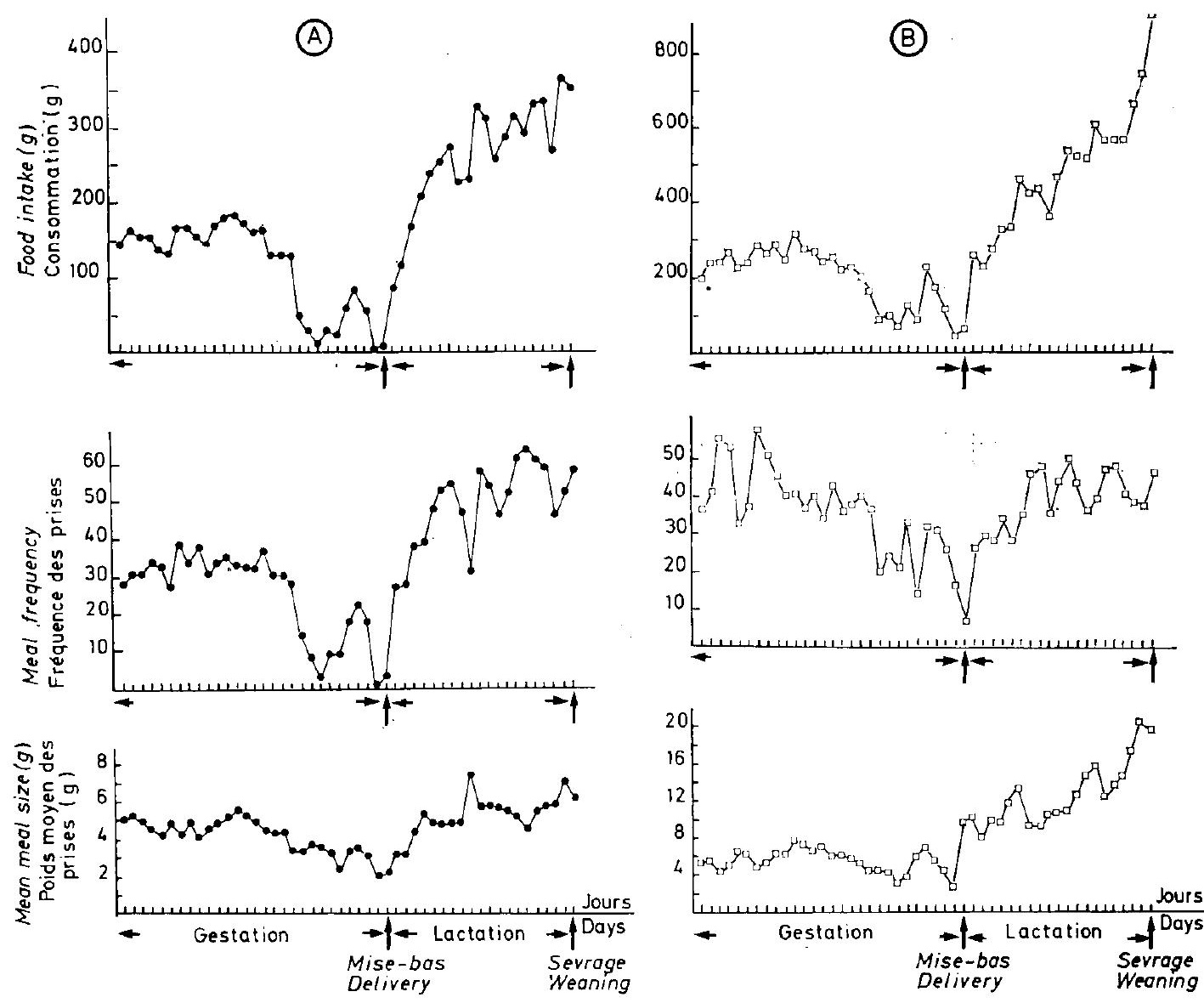

FIG. 2. - Évolution des caractéristiques de l'ingestion solide (A) et liquide (B)

chez la lapine gestante puis allaitante : exemple d'une lapine.

Evolution of solid (A) and liquid (B) food intake characteristics during pregnancy and lactation: example for one doe rabbit. 
téristiques de consommation. Nous avons mis en parallèle pour une lapine 1'évolution au cours de la gestation et de la lactation des consommations, nombre de prises et poids moyen des prises solides et liquides. Compte tenu des échelles adoptées on peut noter un parallélisme presque parfait entre l'évolution dans le temps des quaritités d'aliment solide ingérées et du nombre de prises. Ce phénomène est moins marqué et beaucoup plus variable dans le cas de l'eau de boisson où la régulation de l'ingestion semble se faire surtout par la variation du poids moyen des prises.

L'étude de l'évolution des fréquences et du poids moyen des prises au cours des mises bas successives ne permet pas de déterminer quel est le facteur qui explique le plus les différences de niveau de consommation enregistrées. Dans le cas de l'aliment, l'augmentation de la fréquence des prises par rapport au cycle gestation-lactation précédent, semble avoir cependant une place prépondérante en fin de gestation et en fin de lactation. Dans le cas de l'eau de boisson, l'augmentation de consommation au cours des mises bas successives peut être dûe selon les lapines soit à l'augmentation du poids moyen des prises, soit à celle de la fréquence. Il est difficile de dégager une règle générale.

Dans le tableau I sont reportées, semaine par semaine, les valeurs moyennes journalières \pm écart-type des quantités, fréquences et poids moyen des prises d'aliment et d'eau de boisson. Les calculs ont été effectués pour les trois semaines de lactation ( 2 I jours) et pour les quatre semaines précédant la mise bas ( 28 jours) appelées par commodité : première, deuxième, troisième et quatrième semaine de gestation. Nous retrouvons les tendances précédemment décrites.

\section{Quantités ingérées}

Au début de la gestation, les consommations présentent un plateau, les lapines ingérant en moyenne I $50 \mathrm{~g}$ d'aliment et $290 \mathrm{~g}$ d'eau de boisson par jour. La consommation d'aliment diminue dès la troisième semaine de gestation et n'est plus que de $90 \mathrm{~g}$ par jour lors de la quatrième semaine.

La consommation moyenne d'eau de boisson diminue plus tardivement (de 30 p. cent environ à la quatrième semaine de gestation). Après la mise bas, les consommations augmentent fortement puisqu'elles passent à $2 \mathrm{I} 5 \mathrm{~g}$ par jour pour l'aliment et $385 \mathrm{~g}$ pour l'eau de boisson. Ces valeurs croissent de la première à la troisième semaine de lactation. Exxprimée en valeur relative par rapport au plateau de consommation, l'augmentation est plus forte en fin de lactation pour l'aliment que pour l'eau de boisson.

\section{Fréquence des prises}

En début de gestation, les lapines effectuent en moyenne 30 repas solides par jour. La fréquence des repas diminue la semaine précédant la mise bas puis augmente très rapidement et semble atteindre un plateau dès la deuxième semaine de lactation aux environs de 52-54 prises par jour. Dans le cas de l'eau de boisson la fréquence des prises varie relativement peu : 30 par jour en moyenne durant la gestation, 39 par jour en moyenne durant la lactation. Ainsi, durant la gestation, le nombre de prises d'aliment liquide est voisin du nombre de prises d'aliment solide. Fin fin de lactation au contraire, la fréquence des repas liquides est inférieure $(37-39)$ à celle des repas solides $(52-54)$. 


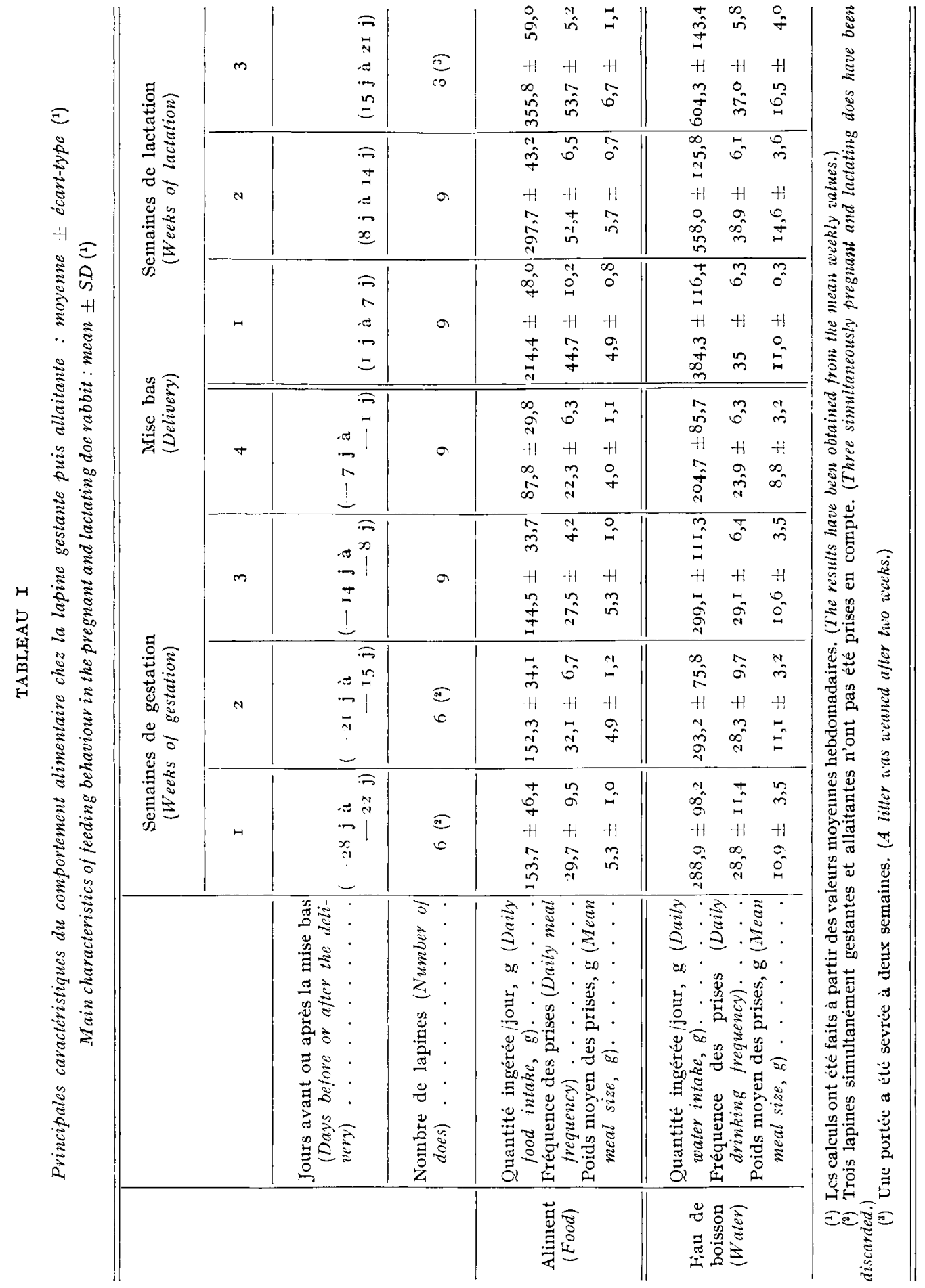


Poids moyen des prises

Le poids moyen des prises d'aliment solide est d'environ $5 \mathrm{~g}$ et varie relativement peu tout au long de la gestation et de la lactation. Il a cependant une légère tendance à augmenter en fin de lactation. Dans le cas de l'eau de boisson le poids moyen des prises varie davantage et en particulier augmente de manière importante au cours de la lactation.

D'une manière générale, la variabilité des résultats est importante, particuliè-
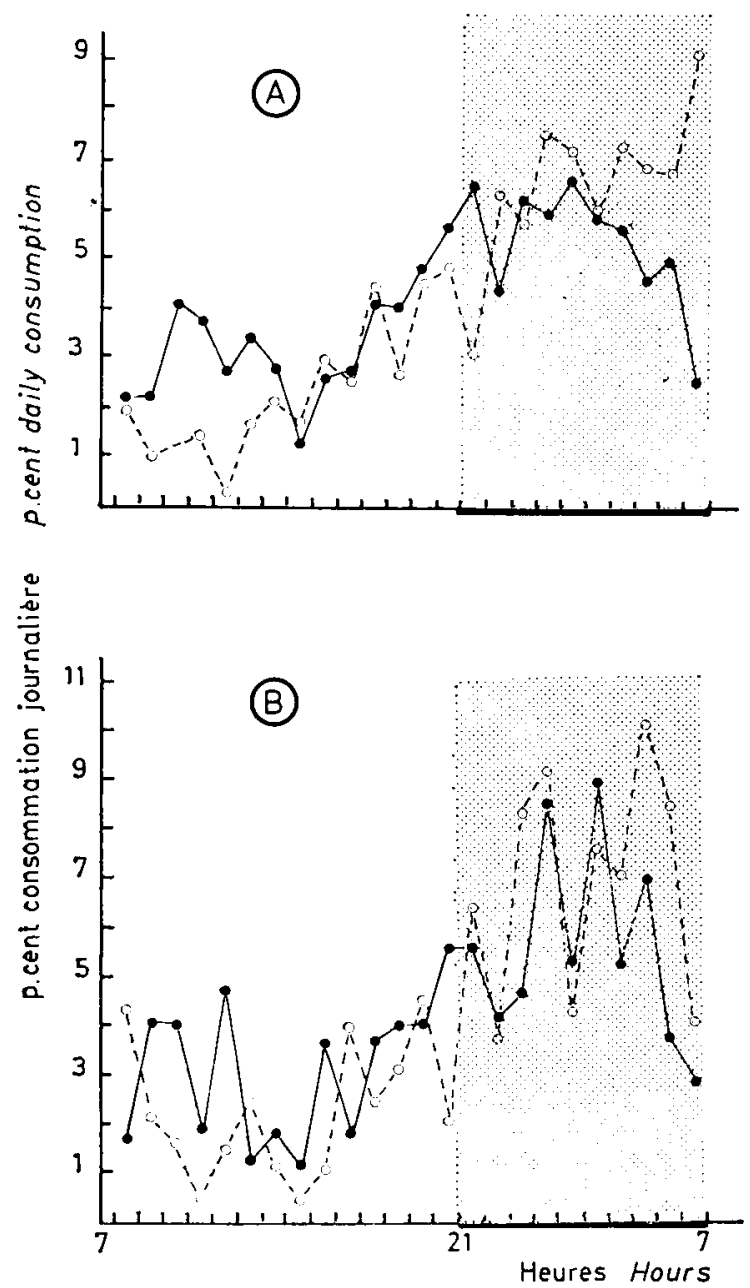

FIG. 3. - Variations nycthémérales de la consommation relative d'aliment (A et d'eau de boisson (B) pendant la gestation :exemple d'une lapine.

Circadian distribution of food intake (A)

and water intake (B) during pregnancy: example for one animal.

- - - - $\mathrm{I}^{\mathrm{re}}$ semaine de gestation ( $\mathrm{s}$ st week of pregnancy)

$\div 4^{\mathrm{e}}$ semaine de gestation (4th reee of pregnancy).

La zone ombrée correspond à la période d'obscurité.

The shaded areas correspond to the dark period. 
rement dans le cas de l'eau de boisson, ce qui peut être dût en partie à une moindre précision des mesures dans le cas de l'eau du fait de gaspillages possibles et variables suivant les lapines. Les différences entre individus ont tendance à diminuer quand on passe de la gestation à la lactation.

\section{2. -- Répartition de la consommation au cours du nycthémère}

Nous avons représenté chez une lapine gestante l'évolution des consommations relatives d'aliment et d'eau de boisson pendant le nycthémère (fig. 3).

Bien que les niveaux de consommation soient très différents, l'étude comparée,
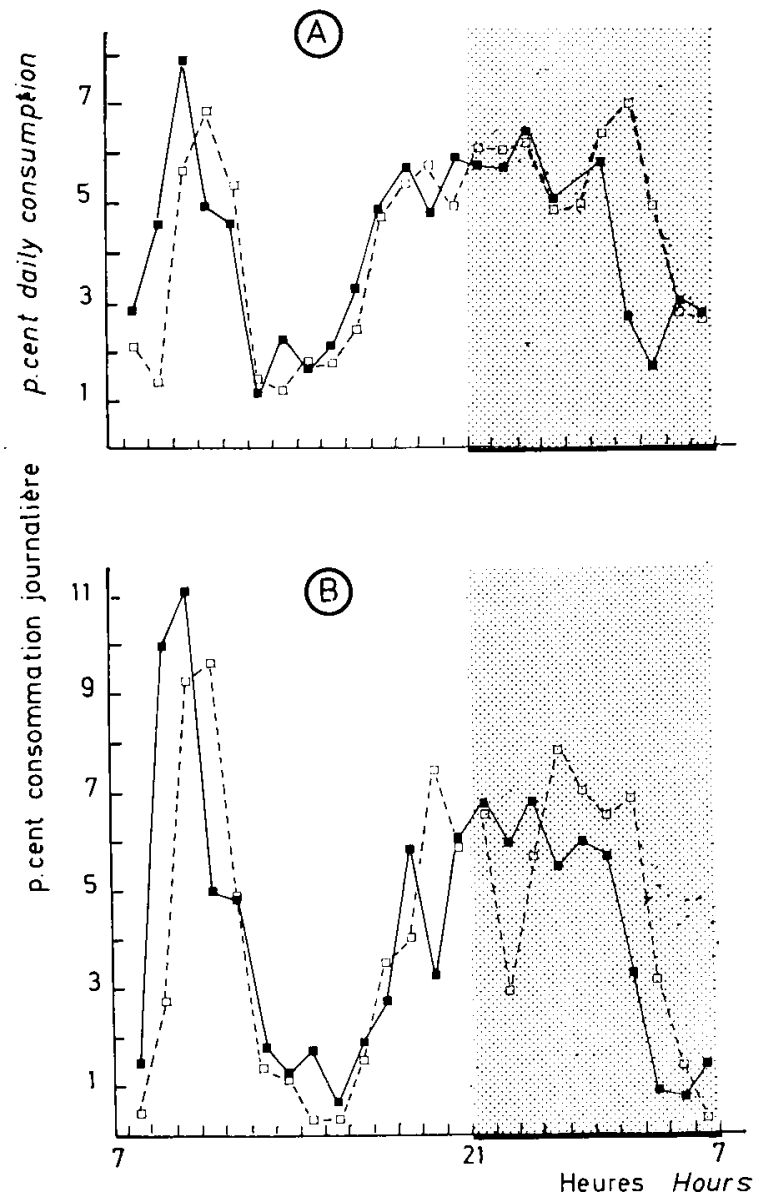

FIG. 4. - Variations nycthémérales de la consommation relative d'aliment (A) et d'eau de boisson (B) pendant la lactation : exemple d'une lapine en allaitement contrôlé. Circadian distribution of food intake (A)

and water intake (B) during lactation: example for a rabbit doe of the controlled suckling lot.

- I I ${ }^{\text {re }}$ semaine de lactation (Ist week of lactation).

- $3^{\mathrm{e}}$ semaine de lactation ( 3 rd week of lactation).

La zone ombrée correspond à la période d'obscurité.

The shaded areas correspond to the dark period. 
pour les différentes lapines, de la répartition des consommations durant la première et la quatrième semaine de gestation ne fait pas apparaître de différences systématiques. Le seul caractère réellement constant est la consommation nocturne largement prédominante, les quantités ingérées atteignant un maximum à des heures variables au cours de la période d'obscurité.

Chez les lapines en " allaitement contrôlé " (fig. 4) la consommation nocturne reste prédominante, mais la période de forte consommiation d'aliment et d'eau de boisson commence vers $\mathrm{I} 7$-I 8 heures, soit bien avant l'extinction de la lumière. La consommation est faible en fin de nuit.

La répartition nycthémérale de la consommation est fortement modifiée par la tétée à heure fixe. Une quantité importante de nourriture et d'eau est ainsi

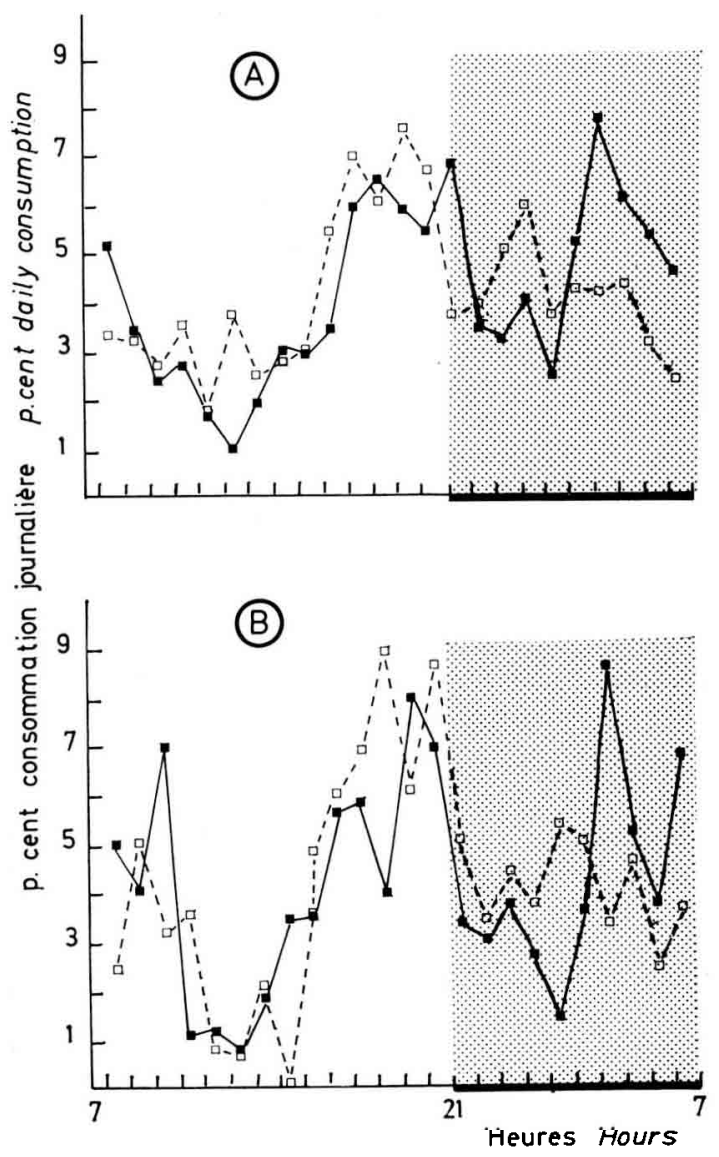

FIG. 5. - Variations nycthémérales de la consommation velative d'aliment (A) et d'eau de boisson (B) pendant la lactation : exemple d'une lapine en allaitement libre. Circadian distribution of food intake (A)

and water intake (B) during lactation: example for a doe rabbit of the free suckling lot.

- I $^{\mathrm{ro}}$ semaine de lactation (Ist week of lactation).

La zone ombrée correspond à la période d'obscurité.

The shaded areas correspond to the dark period. 
ingérée entre 9 et II heures, il faut noter également que les lapines boivent parfois en anticipant sur l'heure de la tétée.

On n'observe aucune évolution dans la répartition nycthémérale des consommations solide et liquide entre la première et la troisième semaine de lactation ou dans la répartition horaire pour deux lactations successives.

Chez les lapines en " allaitement libre ", les courbes de consommation relative (fig. 5) ne présentent pas un pic analogue à celui observé précédemment lors de la tétée à heure fixe. Il en est de même si on analyse les courbes individuelles de consommation jour par jour. La répartition horaire des consommations d'aliment et d'eau est beaucoup plus variable que dans le cas précédent.

\section{3. - Principaux paramètres zootechniques et liaisons entre production laitière et consommation d'eau et d'aliment}

Le tableau 2 montre l'évolution au cours du temps de quelques paramètres zootechniques. Dans le cas d'un sevrage des lapereaux à trois semaines, la production laitière des lapines et la quantité de lait reçue par lapereau augmentent jusqu'au sevrage. Par contre, l'utilisation du lait est de moins en moins bonne. Ia variabilité des résultats est très importante.

\section{TABLEAU 2}

Evolution au cours du temps de la quantité de lait produite par lapine, de l'indice de consommation des lapereaux et du lait consommé par lapercau : moyennes \pm écart-type

Pattern of doe milk production, milk efficiency ratio and milk consumed by the young rabbit: mean $\pm S D$

\begin{tabular}{|c|c|c|c|}
\hline Semaines (Weehs) . . . . . . . & I & 2 & 3 \\
\hline $\begin{array}{c}\text { Nombre de lapines (Number of } \\
\text { does) } . . . \\
\end{array}$ & 9 & 9 & 8 \\
\hline $\begin{array}{l}\text { Lait produit/lapine, g/semaine } \\
\text { (Milk produced//emale rabbit, } \\
\text { g/week) . . . . . . . . . . }\end{array}$ & $800 \pm 192$ & I $287 \pm 29$ I & I $555 \pm 245$ \\
\hline $\begin{array}{l}\mathrm{g} \text { de lait } / \mathrm{g} \text { de gain de poids ( } g \text { of } \\
\text { milk needed for one } g \text { of weight } \\
\text { gain)............. }\end{array}$ & $1,66 \pm 0,29$ & $\mathrm{I}, 84 \pm 0, \mathrm{I} 5$ & $\mathrm{~J}, 92 \pm 0, \mathrm{I} 7$ \\
\hline $\begin{array}{l}\text { I.ait consomme /lapereau, } \mathrm{g} / \mathrm{se}- \\
\text { maine (1) (Milk consumed by the } \\
\text { young rabbit, g/week). . . . . }\end{array}$ & I 23 & 198 & 219 \\
\hline
\end{tabular}

(1) Moyennes établies en tenant compte des effectifs différents de chaque portée. (Mcans established, taking account of the different number of young rabbits in each litter.) 
Le tableat 3 donne les coefficients de corrélation calculés entre quantité de lait produite par jour et quantités d'aliment et d'eau ingérées par jour. Ces corrélations sont élevées et semblent d'autant meilleures que la production par lactation est plus importante. Il existe aussi une corrélation significative entre la quantité de lait produite par jour et les quantités d'aliment et d'eau ingérées dans les deux heures qui suivent la tétée mais elle est due surtout à l'influence de la variable : quantité d'aliment solide ou liquide ingérée en 24 heures. Le coefficient de corrélation partielle éliminant 1'influence de cette variable est en effet pratiquement nul.

\section{TABLEAU 3}

Coefficients de corrélation entre quantité de lait produite par jour $(y)$ et consommation solide et liquide par jour $(x)$

Corvelations between amounts of daily milk production $(y)$ and daily food and water intake $(x)$

\begin{tabular}{|c|c|c|c|c|}
\hline & & $\begin{array}{c}\text { Production laitière } \\
\text { totale estimée, g } \\
\text { (Total amount of milk } \\
\text { produced by female } \\
\text { rabbit, g) }\end{array}$ & $\begin{array}{l}\text { Quantité d'aliment } \\
\text { ingérée par jour } \\
\text { (Daily food intake) } \\
\mathbf{r}_{\mathrm{xy}}\end{array}$ & $\begin{array}{c}\text { Quantité d'eau de } \\
\text { boisson ingérée par } \\
\text { jour } \\
\text { (Daily water intake) } \\
{ }^{r_{x y}}\end{array}$ \\
\hline $\begin{array}{c}\text { Lapine I } \\
(\text { Doe rabbit I) }\end{array}$ & $\begin{array}{l}\text { Ire mise bas } \\
\text { (Ist delivery) } \\
2^{\mathrm{c}} \text { mise bas } \\
\text { (and delivery) }\end{array}$ & $\begin{array}{l}3160 \\
43^{80}\end{array}$ & $\begin{array}{l}0,87 \\
0,87\end{array}$ & $\begin{array}{l}0,77 \\
0,63\end{array}$ \\
\hline $\begin{array}{c}\text { Lapine } 2 \\
\text { (Doe rabbit } 2 \text { ) }\end{array}$ & $\begin{array}{l}\text { I }^{\mathrm{re}} \text { mise bas } \\
\text { (Ist delivery) } \\
2^{\mathrm{e}} \text { mise bas } \\
\text { (2nd delivery) }\end{array}$ & $\begin{array}{l}3410 \\
\text { I } 314\end{array}$ & $\begin{array}{l}0,93 \\
0,69\end{array}$ & $\begin{array}{l}0,9 \mathrm{I} \\
0,74\end{array}$ \\
\hline $\begin{array}{c}\text { Lapine } 3 \\
\text { (Doe vabbit } 3 \text { ) }\end{array}$ & $\begin{array}{l}\text { Ie mise bas } \\
(\text { Ist delivery) } \\
2^{\circ} \text { mise bas } \\
\text { (2nd delivery) }\end{array}$. & $\begin{array}{l}2810 \\
4155\end{array}$ & $\begin{array}{l}0,85 \\
0,91\end{array}$ & $\begin{array}{l}0,53 \\
0,86\end{array}$ \\
\hline $\begin{array}{c}\text { Lapine } 4 \\
\text { (Doe rabbit } 4 \text { ) }\end{array}$ & $\begin{array}{l}\text { I }^{\mathrm{re}} \text { mise bas } \\
(\text { Ist delivery) } \\
2^{\mathrm{C}} \text { mise bas } \\
2 \text { nd delivery }) \\
3^{\mathrm{o}} \text { mise bas } \\
\text { (3rd delivery) }\end{array}$ & $\begin{array}{l}4330 \\
4 \text { II } 8\end{array}$ & $\begin{array}{l}0,95 \\
0,91\end{array}$ & $\begin{array}{l}0,67 \\
0,88 \\
0,74\end{array}$ \\
\hline
\end{tabular}

\section{Discussion}

La forme des courbes individuelles de consommation d'aliment rappelle celle de la courbe moyenne observée par LEBAS (I97I) pour des animaux de race californienne. Il faut noter en particulier l'arrêt presque total de consommation d'aliment et d'eau de boisson la veille ou l'avant-veille de la mise bas. L'existence d'un 
pic de consommation vers le quatrième jour avant la mise bas n'a pas été signalé jusqu'à présent à notre connaissance. Ce phénomène demande toutefois à être confirmé sur un plus grand nombre d'animaux.

Les courbes de consommation d'aliment et d'eau de boisson pendant la lactation sont d'autre part très voisines de la courbe de production laitière telle qu'elle a été décrite par LEBAS (I968) pour des lapines de race Fauve de Bourgogne.

$\mathrm{Au}$ cours de la gestation, l'évolution des consommations relatives d'aliment et d'eau de boisson pendant le nycthémère rappelle celle observée par CHERUBIN (I974) puis PRUD'HON et al. (I975a) sur des lapins en croissance, par SANDERSON et VANDERWEEI,E (I974), HöRNICKE et al. (I976) sur des animaux adultes.

Les modifications très rapides des consommations journalières d'aliment et d'eau immédiatement après la mise bas et au cours de la lactation se répercutent sur la répartition horaire. Dans le cas des lapins en "allaitement libre " la consommation semble se répartir sur l'ensemble du nycthémère, la consommation nocturne étant moins accentuée que dans le cas des lapines en "allaitement contrôlé ". Tout se passe comme si la tétée à heure fixe regroupait les consommations sur deux périodes distinctes, la courbe présentant alors une allure biphasique.

Dans les deux cas, la période de forte consommation d'aliment et d'eau de boisson commence vers $\mathrm{x} 7$-I 8 heures, soit bien avant l'extinction de la lumière, la consommation étant faible en fin de nuit. Ceci est à rapprocher des résultats de PRUD'HON et al. (I975a et I976) sur lapins en croissance ou soumis à différentes températures. Dans ces différents cas, il semble que les modifications des caractéristiques de consommation portent avant tout sur les périodes précédant ou succédant à l'extinction des feux.

Nos observations sur les lapines en allaitement contrôlé ont mis en évidence l'existence d'un pic de consommation dans les deux heures qui suivent la tétée. On pourrait penser que ce pic correspond à un ajustement à court terme des dépenses d'énergie et d'eau engendrées par l'allaitement. En fait, il ne semble pas exister de relation directe entre la quantité de lait produite et les quantités d'aliment ou d'eau ingérées durant ce laps de temps comme le montre la faible valeur des coefficients de corrélation partielle.

Par ailleurs, chez les lapines en allaitement libre, nous n'avons pu mettre en evidence un phénomene semblable. Il est d'ailleurs probable que si une forte consommation a lieu dans ce cas à la suite de l'allaitement, elle est masquée par le fait que les tétées sont généralement nocturnes et que 1'horaire en est variable comme le note YARDIN (I968). Il est aussi possible que le pic de consommation observé après la tétée đans le cas de 1'allaitement contrôlé soit artificiel. On peut imaginer que dans l'attente de la tétée, la lapine cesse toute consommation et se remette à consommer seulement après l'allaitement.

Accepté pour publication en janvier 1978 .

\section{Summary}

\section{Solid and liquid feed intake characteristics in pregnant and lactating doe-rabbits fed ad libitum}

The number, length and circadian distribution of solid and liquid meals were recorded graphically in pregnant and lactating doe-rabbits. The recording device was previously described by PRUD'HON et al. (1972). Two lots of 4 and 7 white New Zealand doe-rabbits were studied during 9 and 8 gestation-lactation cycles. 
In the first lot (controlled suckling) the young were separated from their datns and allowed to suck once a day. The amounts of milk produced per rabbit were estimated every day by the weight loss of the doe during suckling (LFBAs, I968).

In the second lot (free suckling) the does had free access to the nest.

This study gives a precise description of the variation in the solid and liquid feed intake characteristics (number of meals, length, quantity) during pregnancy, lactation and for several consecutive gestation-lactation cycles.

Beside the well known variation in feed intake and milk production during the first three weeks of lactation, we observed:

- an almost total stop of feed and water intake the day before parturition,

- a predominantly nocturnal intake pattern in the pregnant doe, independently of the stage of gestation, lactation.

- a change in the circadian distribution of feed and water intake with the beginning of

In controlled suckling does a biphasic intake pattern was observed. The maximal values were located after suckling and during the few hours following or preceding light extinction.

In free suckling does, both the diurnal and nocturnal intake patterns were more variable.

Moreover, the increase in feed intake observed in lactating does seemed mainly to be due to an increase in the frequency of solid meal intake and to a less extent to an increase in their size during late lactation. This increase was more variable and less obvious for the water intake pattern where the variation of the meal size played a more important part.

Correlations between milk production and feed intake during the 24 h-cycle were large On the contrary, the partial correlation coefficients between milk production and amount of feed consumed during the two hours after suckling were very low. This suggests that the restoring of the energy and water expenditure due to lactation takes 24 hours or more.

\section{Références bibliographiques}

Chertisin M., r974. Étude du comportement alimentaire du Lapin domestique du sevrage à la dix-huitième semaine. Influence de quelques facteurs extrinsèques sur les paramètres de l'ingestion alimentaire. Thèse Doctorat Spécialité, Université des Sciences et Techniques alu Languedoc, Montpellier, $5^{8} \mathrm{p}$.

Chevilitard L., Gasnier A., Maypr A., I939. Les modalités de 1'ingestion chez les lapins qui s'alimentent spontanément. Ann. Physiol. Physicochim. Biol., 15, 271-281.

Hörnicke H., Dietzel F., AIgNer E., Scirulte R., I976. Alimentary behaviour in rabbits under different influences. I ${ }^{\text {er }}$ Congrès international cunicole. Dijon (France). A.S.F.C. Communication, $\mathrm{n}^{\circ} \mathrm{I} 3,4 \mathrm{p}$.

LEBAS F., rg68. Mesure quantitative de la production laitière chez la lapine. Ann. Zootech, 17, $169-182$.

LEBAS F., I97I. Le Lapin de chair. Ses besoins nutritionnels et son alimentation pratique. Supplément aux nouvelles de l'Aviculture, $\mathrm{n}^{\circ} \mathrm{I} 53,35 \mathrm{p}$.

Prud'hon M., Carles Y., Goussopoulos J., Koehi P. F., i972. Enregistrement graphique des consommations d'aliments solide et liquide du Lapin domestique nourri ad libitun. Ann. Zootech., 21, 45I-460.

Prud'hon M., Cherrubin Michaelle, Goussoponlos J., Carles Y., 1975a. Évolution, au cours de la croissance, des caractéristiques de la consommation d'aliments solide et liquide du Lapin domestique nourri ad libitum. Ann. Zootech., 24, z89-298.

Prud'hon M., Cherubix Michaelle, Carles Y., Goussopoulos J., 1975b. Effets de différents niveaux de restriction hydrique sur l'ingestion d'aliments solides par le Lapin. Ann. Zootech., 24, 299-3 Iо.

Prud'hon M., I976. Comportement alimentaire du Lapin soumis aux températures de Io, 20 et $30^{\circ} \mathrm{C}$. $I^{e r}$ Congrès international cunicole, Dijon (France). A.S.F.C., Communication $\mathbf{n}^{0} \mathbf{I}_{4}$, $6 \mathrm{p}$.

Sandersox J., VANDERWEELE D., 1975. Analysis of feeding patterns in normal and vagotomized rabbits. Physiology and behaviour, 15, 357-364.

YARDIX M., I968. Quelques aspects qualitatifs et quantitatifs de l'activité de la lapine allaitante. Rev. comp. Animal., 2, 72-76.

Zarrow M. X., Denenberg V., Anderson C., 1965. Rabbit frequency of suckling in the Pup. Science, 150, I835-1 836 . 